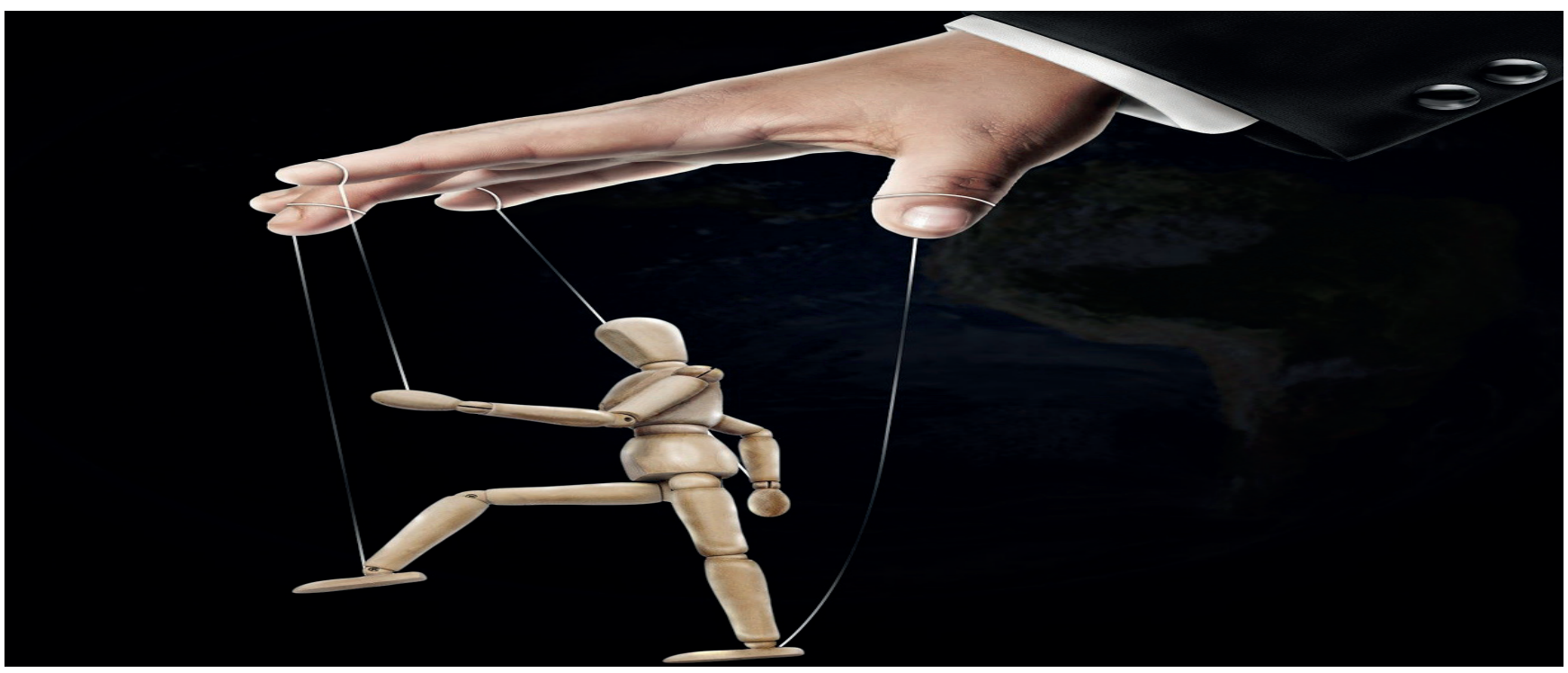

Tomado de: https://pixabay.com/es/photos/mano-marioneta-mu\%C3\%B1eco-de-nieve-784077/

\title{
Los valores como presupuesto en el desarrollo de políticas públicas en el combate a la corrupción en México en el marco de la "nueva normalidad"
}

\section{Values as a budget in the development of public policies in the fight against corruption in Mexico within the framework of the "new normality"}

\author{
Luis Alberto Osornio-Saldívar*, Eduardo Martínez-Altamirano, Idalia Acosta-Castillo
}

\section{Resumen}

México está viviendo un periodo de transición de un sistema obsoleto e inoperante para nuestro tiempo a otro totalmente nuevo. Es un proceso de cambio que entraña riesgos y resistencias, pero que es necesario e impostergable, pues su retraso o cancelación implicaría altos costos para el futuro de una nación en plena transformación, de tal manera que el análisis del Sistema Nacional Anticorrupción debe partir de un fundamento ético, mismo que expondremos a continuación, que es de donde deben partir, precisamente, las políticas públicas en materia de seguridad pública y combate a la corrupción como parte de ese reto hacia la nueva normalidad.

Palabras clave: nueva normalidad, sistema nacional anticorrupción, ética, valores, política, corrupción

\section{Abstract}

Mexico is living the transition from an old and obsolete system to an entirely new one. It is a process of change that finds strengths of resistance and also risks, but it is necessary and imminent. Its delay or cancellation would imply high costs for the future of the nation that seeks change, therefore the analysis of the New National Anticorruption System must start of the ethical foundation where the public policy on security matters lies as a challenge to the new normal.

Keywords: new normality, national anticorruption system, ethics, values, politics, corruption

*Fecha de recepción: 10/09/2020 Fecha de aceptación: 30/09/2020 Correspondencia: luis.osornio@uaslp.mx Dirección: Universidad Autónoma de San Luis Potosí. 


\section{INTRODUCCIÓN}

El trabajo que aquí proponemos, pone especial énfasis en ciertos cambios de fondo que podemos y debemos operar desde una perspectiva cultural y cómo estos pueden materializarse e influir en el desarrollo de las acciones en contra de la corrupción, sobre todo, en el desarrollo de políticas públicas en el marco del Sistema Nacional Anticorrupción. Por ello, proponemos dar inicio a nuestros posicionamientos a partir de un acercamiento o aproximación con ciertas opiniones conceptuales, mismas que dan sustento a nuestras opiniones. Es así que es necesario observar de inicio las opiniones del tratadista, (Jorge, 2010) el cual nos aclara que "La cultura comprende las ideas, valores y hábitos de individuos y grupos referidos al proceso político, sus actores e instituciones" (p.18), en estas ideas cabe resaltar de manera introductoria al tema que una nueva política pública en materia de seguridad debe reenfocarse desde una democracia representativa que combata la corrupción, posea un sentido social que impulse de forma responsable el desarrollo sostenido y contenga, además, un soporte de valores éticos como su fundamento.

De acuerdo con lo anterior, es importante señalar que la clave del cambio generacional en materia de educación y seguridad radica en promover los valores en nuestro sistema educativo, el cual comprende a "las instituciones del gobierno y de la sociedad encargadas de proporcionar servicios educativos y de preservar, transmitir y acrecentar la cultura de los mexicanos" (Subsecretaría de Educación Básica, 2019). La pervivencia de los valores es, sin duda, emergente como política pública, por lo que a educación en los valores resulta algo esencial para el buen logro de una transición que permita el combate efectivo al fenómeno de la corrupción; la cual, en el mejor de sus escenarios, propiciará el progreso que se desea para el país como el que deseamos con desarrollo, democracia, justicia y de oportunidades para todos los mexicanos. Es importante aclarar de inicio que los valores son definidos -en esta aportación académica- como "ejes fundamentales por los que se orienta la vida humana y constituyen a su vez, la clave del comportamiento de las personas" (Sartre, 1994), p. 371. Por ello, se dice que son sustratos humanos y universales, mientras que los valores éticos son parte esencial de la transformación de la política pública y del sistema de educativo y de seguridad que requiere el país, ya que son elementos primordiales de la cultura, a través de los cuales será posible mantener la existencia de una convivencia social armónica. Los académicos y la comunidad en general como integrantes de la sociedad o del "grupo de personas que vive en un determinado territorio, sometido a un sistema común de autoridad política y que es consciente de poseer una identidad que lo distingue de otros grupos" (Giddens, 1999), p. 739, persiguen la búsqueda del bien común a través del cumplimiento institucional del análisis y la promoción de los valores en desarrollo de una política pública en materia de seguridad. Por esta razón, con este estudio pretendemos introducir y captar la atención de los lectores hacia un tema de gran actualidad, mediante el análisis del origen, los conceptos y la exposición de algunas consideraciones o conclusiones iniciales sobre diversos tópicos; primero, en torno a los valores y segundo, que veamos cómo estos son un presupuesto fundamental en el desarrollo de políticas públicas en contra del lamentable fenómeno de la corrupción.

Por último, como parte de esta nota introductoria, es importante considerar que la normalidad como definición es la cualidad o condición de normal que se ajusta a las normas y que se ajusta a valores medios. Y la normalidad de un comportamiento está vinculada a la conducta de un sujeto que no muestra diferencias significativas respecto a la conducta del resto de su comunidad. Por ello, vale la pena aquí que nos preguntemos ¿qué es lo más normal, que los políticos sean honestos o corruptos? La respuesta la dejamos a su consideración, pero estamos seguros que las siguientes líneas ayudarán a vislumbrar posicionamientos más justos que incidan en el fortalecimiento de nuestro Estado de Derecho. 


\section{Problemática}

Los problemas que enfrenta el país en la actualidad son, por mucho, más complejos y diversos que en el pasado, por lo que el estado actual de las cosas no debe reducirse a la aplicación de soluciones tradicionales o enfoques extrínsecos, ya que los problemas públicos tienen profundas raíces humanas, entre las que destaca la libertad como aquella capacidad en donde se origina la moralidad de sus actos.

Es necesario admitir que existe una causa que se encuentra más allá de los síntomas que se pretenden atacar, la cual radica en que padecemos una crisis moral. El abandono del criterio moral, el cual según (Morales, 2017) es el "conocimiento suficiente para regular nuestras costumbres, o tan grande como la que tenemos acerca de las cosas que no tenemos costumbre de dudar en relación con la conducta de vida, aun sabiendo que puede ser que, absolutamente hablando, sean falsas" (p.17), por parte de las personas al decidir sobre sus actos. En parte, porque se prefieren otros criterios, como el tener más bienes materiales o mayor poder, sacrificando el ser mejor.

Sin caer en posturas relativistas o subjetivistas, es evidente que la sociedad necesita ponerse de acuerdo sobre cuáles valores básicos permitirán convivir y poder confiar en las personas, en su palabra, en que seremos respetados en nuestros derechos y persona sin tener que acudir para ello necesariamente a la fuerza de la ley.

La norma positiva lato sensu es un elemento social presente en la sociedad para garantizar el orden, pero al igual que la justicia es "característica posible más no necesaria del orden social, una virtud del individuo pues un hombre es justo cuando su obrar concuerda con el orden considerado justo" (Kelsen, 2000, p. 5).

Debe tomarse en cuenta que en diversas ocasiones lo que motiva que los ciudadanos trabajen por el bien común en lugar de trasgredir la ley, no es el temor a la sanción de la justi- cia humana, sino la convicción y vivencia de ciertos valores como la solidaridad, la subsidiariedad o el patriotismo. Si bien es cierto que los valores éticos se forman en la institución social de la familia la cual es un "conjunto de elementos ligados entre sí por reglas de comportamiento y por funciones dinámicas en constante interacción interna y con el exterior" (Valladares, 2008, p. 4), también lo es que la crisis moral que enfrentamos requiere que se enfatice su promoción tanto en ella como en la escuela e incluso en el resto de los ámbitos de la vida social.

Afortunadamente, son todavía muchas las familias y personas las que en nuestra sociedad constituyen su reserva moral por la forma en que conducen sus vidas conforme a los más nobles valores; de otra manera no habría esperanza de mejoramiento. No obstante, es preciso trabajar para que este ejemplo se extienda y exista el deseo de una mejora continua personal y social en todos los sectores.

Para impulsar el desarrollo social y económico del país se tienen que atacar diversas actitudes y antivalores propios de la cultura de forma íntegra, propiciando un enfoque holístico de la cultura, que permita analizar todos los elementos que le corresponden por naturaleza con todos los por XXX y no como un conjunto de aspectos inconexos.

El ser humano tiene una dignidad ontológica la cual -desde una perspectiva teórico-doctrinal- "es la rama de la filosofía que se dedica a reflexionar sobre los modos esenciales de existencia de las cosas" (Posadas, 2014), p. 4, además, posee ética que según (Parker, 2001) "es el uso de herramientas de la razón para generar reglas que proporcionen un juicio en circunstancias tanto generales como particulares". La ontológica es inherente a la naturaleza humana, es decir, se posee por el hecho de ser hombre, por ello, presenta las siguientes características:

- Original: empieza desde el nacimiento.

- Gratuita: no se necesita ningún mérito para obtenerla. 
- Imperdible: la persona siempre la poseerá.

- Incomunicable: cada persona la posee.

La dignidad ética se deriva del comportamiento y la conducta de la persona y de ella depende su crecimiento o no, esto porque la construye con sus actos y por tanto posee las siguientes características:

- Dinamismo: porque se construye mediante los actos humanos.

- Crecimiento o decrecimiento: hay personas que tienen mayor dignidad que otras.

- Posibilidad de perderla: se puede ser indigno.

Con fundamento en lo antes manifestado, queda claro que la persona, aunque única e irrepetible, por su común naturaleza racional, pertenece a la sociedad humana, tiene con ellos un vínculo y, por tanto, es un ser social. Además, por su doble capacidad de conocer y querer, puede abrirse y establecer relaciones con el mundo que le rodea, ya sea el espacio físico, personal y el de la trascendencia.

Ante la extinción de dichas realidades, la persona tiene una infinita gama de posibilidades de elección para conocer y querer. Esa capacidad o poder de elección supone que la voluntad puede auto determinarse, y ahí se basa y origina la libertad de la persona.

Gracias a la libertad, el hombre se gobierna y se posee a sí mismo, dirigiendo sus acciones hacia distintos bienes y haciendo una elección de entre ellos, esto es lo que en gran medida determina su modo de ser personal.

Lo anterior lleva a la consideración de que la persona se constituye con sus acciones, y que, partiendo de su humanidad y en relación con los otros y con el entorno, libre y responsablemente, conduce su vida de determinada manera. Esto supone necesariamente que el hombre es un ser inacabado, que debe perfeccionarse, ya que de lo contrario no cabría en él ningún tipo de crecimiento.

Bajo esta lógica, encontramos que lo propio en el hombre es el desarrollo de sus capa- cidades en orden, al respecto (Alles, 2008) menciona "Que la capacidad o capacidades, es la aptitud con que cuenta cualquier persona para llevar acabo Cierta tarea. Es decir que todos los seres humanos estamos capacitados para realizar con éxito cualquier tarea" (p. XXX).

Cuando el hombre dirige libremente sus acciones hacia la verdad y el bien, éste se realiza como persona feliz. De esta forma comprende que las acciones del hombre y su actuar consciente hacen de él quien realmente es. Para que una acción conduzca al hombre a su perfeccionamiento, este debe ir conforme al verdadero bien de su naturaleza. De este modo, lo ético consistirá en la búsqueda habitual del perfeccionamiento de la persona humana atendiendo a su naturaleza y a sus rasgos característicos.

La ética es una ciencia teórico-práctica y filosófica-normativa encargada del estudio de los aspectos individuales y sociales de la persona y el temor de la moralidad de los actos humanos. Por ello, para objeto de este análisis se puede considerar a la ética y los valores como ciencias formativas, que pueden estructurarse en política pública con enfoque de seguridad para la nación. Así, es imprescindible que el gobierno mexicano dirija con premura esfuerzos del sistema educativo e instituciones conexas hacia la normalización de la cultura en el combate a la corrupción.

\section{Conceptos generales}

¿Qué son los valores? Actualmente escuchamos con bastante frecuencia que los problemas de nuestra sociedad o de la empresa, son atribuidos a la "falta o ausencia de valores". Pero en realidad las personas no saben qué son estos, ni cómo concretarlos en la cotidianidad. Por tal motivo, es necesario definir claramente en qué consisten los multicitados valores, con el objetivo de que estos constituyan un soporte fundamental para la acción con contenido ético.

El concepto etimológico de valor es un término 
que según (Platón, 2013) no es unívoco, sino que es análogo, por lo cual puede ser utilizado como sinónimo de historia, étimo, ético (siempre que tenga un significado distinto), este término deriva del latín valeo-ui el cual está vinculado con la palabra valere, que significa ser fuerte, robusto, prevalecer, tener fuerza, poder y eficiencia. En esta concepción hay un deseo por extraer de la realidad una presencia que se intuye: la fuerza, la potencia de un ser. De esta forma puede definirse el valor como toda perfección real o posible que procede de la naturaleza y que se apoya tanto en el ser como en la razón de ser da cada individuo. El valor como perfección merece la estima, reconocimiento y agrado para cualquier toma de decisiones, puesto que, en ellas influye los valores, su jerarquía y jerarquización.

La jerarquía, como una "forma de organización de diversos elementos de un determinado sistema, en el que cada uno es subordinado del elemento posicionado inmediatamente por encima” (Nunes, 2015), p. 01, está dada por los distintos tipos de sujetos. Cada uno de estos sujetos tiene un grado de perfección y una tarea dentro del conjunto armónico de la naturaleza. Por ello, para tener una jerarquía de valores acorde con lo real, las personas deben conocer los distintos grados de perfección de los seres y adecuarse a lo que cada naturaleza ofrece, ya que, la jerarquía comprende el orden o los grados entre personas, animales, vegetales y cosas.

El contenido de una jerarquía se encuentra ya dado, puesto que se apoya en la perfección o la tarea que desempeñan cada uno de los sujetos (componentes objetivos). Se apoya, por tanto, en algo evidente para todos, que no depende de una apreciación personal, de esta forma sólo hay una verdadera jerarquía de valores, pues sólo existe una jerarquía en los seres humanos. Es así que al ser humano le corresponde conocerla y asumirla tal como es, ya que no es su papel modificarla ni crear nada nuevo. En cambio, la jerarquización es una actividad exclusiva de la persona que consiste en organizar algo de acuerdo con una escala, respetando siempre el orden natural de los seres para elegir, explicar y aplicar sus respectivos valores, de acuerdo con el fin de que persiga. Los valores dan luz a la inteligencia para que pueda guiar a la voluntad, actúan como motivos, como metas hacia las cuales dirigirse. Los valores no bastan para conseguir el perfeccionamiento humano, ya que todo podría quedarse en una simple apreciación que no se hace vida. Así, los valores han de encarnarse en la vida del hombre a través de la realización de actos, convirtiéndose en hábitos, que permitan alcanzar los valores.

Los hábitos según (Aspe y López, 1999) son "el resultado del ejercicio de actividades para poseer un bien o para lograr un fin determinado. Son disposiciones estables que la persona adquiere en la media en que va ejerciendo su libertad. Solo el ser humano es capaz de adquirir hábitos" (p.7). Cuando los hábitos perfeccionan a la persona son llamados virtudes. La virtud es "la disposición que resulta de los mejores movimientos del alma y es también la fuente de las mejores acciones y pasiones de esta" (Aristóteles, 1222), p. 30-32 4-41. No deben confundirse los valores con las virtudes, pues los valores dan luz, muestran la meta e iluminan el camino, pero para llegar a ellos es indispensable la virtud o el actuar bien de forma no espontánea o circunstancial, sino de forma habitual.

No es suficiente valorar, sino que hay que vivir lo valorado, aunque sea más fácil solamente valorar, que vivir lo que se valora. Vivir lo valorado supone ejercitarse hasta alcanzar la virtud. Dado que las virtudes se apoyan en el hábito o disposición de hacer u obrar de forma determinada, vivir lo valorado supone ejercitarse hasta alcanzar la virtud, en consecuencia, si el hábito se perfecciona es una virtud, sino es vicio.

Hay virtudes intelectuales y morales, las primeras perfeccionan a la inteligencia, mientras que las segundas perfeccionan integralmente a la persona. Con las virtudes morales se mejora el hacer, a través de este hacer se mejora el ser, y desde la voluntad que hace se influye 
en la inteligencia que aprende a observar y a reflexionar sobre la vivencia del hacer.

En consecuencia, las virtudes intelectuales propician la bondad del hombre sólo en un determinado aspecto. Las virtudes morales permiten alcanzar la bondad en absoluto porque, la actividad que provocan está apoyada en el ejercicio de las virtudes intelectuales. Las virtudes pertenecen al orden de lo volitivo, pues, aunque las virtudes intelectuales perfeccionan a la inteligencia, el desarrollo de cualquier virtud requiere de la influencia de la voluntad, que sostiene el ejercicio para cimentar el buen hábito. Por ejemplo, el ejercicio de las virtudes intelectuales de quien estudia la posibilidad de un nuevo proyecto para una planta de producción, si lo diseña y lo propone, ejercerá algunas virtudes morales. Los valores estimulan el afán para adquirir o fortalecer los buenos hábitos, de esta forma los valores no pueden reducirse a motivos, puesto que la ausencia de éstos, en el ser humano implicaría no saber el porqué y el para qué de su vida, lo cual conduciría a actuar de forma destructiva y contra su vida, sus prójimos y su medio ambiente.

Los valores revelan ante el hombre el por qué y para qué de la vida, del saber, de la ciencia y la tecnología, esta última según (Bunge, 2018) "es la técnica que emplea conocimiento científico; más precisamente cuerpo de conocimiento es una tecnología en sí y solamente si es compatible con la ciencia coetánea y controlable por el método científico, se lo emplea para controlar, transformar cosas o procesos naturales o sociales". Sin valores o sin una correcta jerarquía de éstos, podemos actuar por el camino fácil, hacia la búsqueda del poder y de la riqueza como únicos fines de la vida, generando así las conocidas secuelas de la corrupción.

La corrupción en los sistemas educativos usualmente deriva de la presencia de culturas organizacionales en las que sistemáticamente se desplazan objetivos institucionales (v. Gr. Lograr una distribución equitativa de oportunidades) para impulsar algunos individuales (usualmente beneficios económicos). Este tipo de cultura y las prácticas que surgen a su amparo, es lo que denominó como "corrupción educacional”, retomando una definición de (Heidenheimer et al.,1989) crímenes e injusticias que hoy vivimos. No basta que en la educación universitaria o en la preparación técnica obtengamos como egresados a excelentes profesionistas o técnicos, necesitamos lograr que los egresados sean sobre todo personas de excelente calidad moral.

Sin embargo, la educación como proceso permanente en el que está inmerso el individuo, no incluye, con la graduación del educando, cierto nivel educativo formal. La persona durante su vida tiene que seguir aprendiendo, creciendo y cambiando, pero la clave está en el proceso en su fase familiar y formal asegure que el conductor del mismo sea ya el propio sujeto, así dependerá de él asimilar y aprovechar adecuadamente las experiencias que su medio ambiente le procure y las propias oportunidades que con su actuar él mismo se otorgue.

Max Scheler, el filósofo de la teoría de los valores, decía que el hombre es el único ser portador de valores. Por ello, ni en la familia, ni en la escuela, ni en la sociedad se deja en el proceso educativo de promover los valores que las personas, que las instituciones y que la cultura representa. Por esta razón se impone una reflexión sobre los valores de que somos portadores y promotores: ¿cuáles son positivos? ¿cuáles son negativos? ¿cuáles conviene promover conforme al concepto que tenemos del hombre y de la sociedad que queremos ser? ¿nuestro actuar promueve los valores que pretendemos?, o, al contrario, ¿existe un divorcio o la contradicción entre lo que queremos y lo que hacemos?

Todas estas reflexiones nos llevan a plantearnos la necesidad de proponernos promover consciente y sistemáticamente ciertos valores que convengan a una mejor convivencia social $\mathrm{y}$ al desarrollo de valores del ser humano en todas sus dimensiones: el amor, la responsabilidad, el respeto, la confianza. 
¿Qué es corrupción? Etimológicamente corrupción proviene del latín corruptio, y significa el acto de romper una unión. La palabra se integra con un prefijo, un verbo y un sufijo. El prefijo con se interpreta como unión o colaboración, el verbo rumpere se traduce como: romper, partir, destrozar, hacer pedazos y estallar; y el sufijo tio implica: acción u efecto.

Gramaticalmente el diccionario de la Real Academia Española (RAE, 2019), define corrupción como el acto de corromper, esta palabra a su vez significa:

1. Tr. Alterar y trastocar la forma de algo.

2. Tr. Echar a perder, depravar, dañar o pudrir algo.

3. Tr. Sobornar a alguien con dadivas o de otra manera.

4. Tr. Pervertir a alguien.

5. Tr. Hacer algo que se deteriore.

6. Tr. Incomodar, fastidiar, irritar. Ar y Nav.

7. Intr. Oler mal. (RAE, 2019).

Bajo esta lógica la palabra corrupción posee una carga negativa, por lo cual, indica la descomposición, perversión o vicio de las características esenciales que componen a un objeto o ente. En su enfoque social, corrupción implica deslealtad o violación de las normas sociales, bajo las que se rige la sociedad. En este orden (Leys,1965) expresa que "la palabra misma (corrupción = cambiar de bueno a malo, degradar, pervertir); denota patrones de acción que derivan su significado del papel de los sistemas de valores en el comportamiento social" (p. 216).

La posición (moralista) que estudia a la corrupción como la perdida de los valores sociales y humanos ha sido criticada en el ámbito académico por posturas racionales (Klitgaard, 2000), modernistas (Weber 1979), y funcionalistas (Leys, 1965), que señalan que la corrupción es un problema más allá de los valores de la sociedad y dirigen el análisis del fenómeno a variables como el desarrollo económico, el análisis racional, el análisis económico como verdaderos mecanismos para comprender y solucionar el problema.
Para estas posturas, el enfoque y conceptos moralistas producen un sesgo emocional que restringe observar los límites entre actos reprochables y actos inmorales, en este sentido (Gonzales, 2003) expresa que "tildar a una persona o acción de corrupta no sirve para tipificar un determinado comportamiento o calificar a una determinada persona, sino que provoca una estigmatización que puede ir más allá de lo razonable" (p. 66).

Para (Garzón, 1997) la corrupción siempre está ligada a un sistema normativo o conjunto de normas que regulan un sistema social, cayendo dentro de este sistema las normas religiosas, jurídicas, económicas, etc. De esta forma la corrupción implica la trasgresión hacia un marco normativo de cualquier especie y no sólo el marco jurídico y la norma positiva. Además de la relación corrupción - sistema normativo, es necesario conocer la distinción entre deber y obligación ya que "el deber caería dentro del ámbito de la moral natural y sería equivalente a deber natural. La obligación, en cambio correspondería al ámbito de la moral adquirida y sería el equivalente a deber posicional” (Garzón, 1997, p. 44).

Bajo la distinción entre deber y obligación se logra observar los límites entre la moral (actos inmorales) y la moral adquirida (actos reprochables), ya que siguiendo (Garzón, 1997) existen deberes posicionales y deberes naturales, los deberes naturales corresponden a la moral natural y se aplican a todos los individuos, mientras que los deberes posicionales corresponden a la moral adquirida, la cual implica que estos se adquieren por voluntad propia y se apliquen a individuos con la facultad de decisión.

De esta forma la aplicación de criterios moralistas no provocan estigmatización en las personas y conductas consideradas corruptas, sino que, permiten generar distinciones entre quienes detentan una posición en el sistema de valores y quienes están obligados a cumplir con él, con el objetivo de crear soluciones al fenómeno. 


\section{¿Qué es Anticorrupción?}

La complejidad del fenómeno y la diversidad de posturas en la academia sobre el significado del concepto corrupción, ha causado que no exista un concepto predominante o una definición ampliamente difundida sobre el significado de anticorrupción, sin embargo, la etimología de la palabra nos permite ver el prefijo "anti" que indica lo opuesto o que lucha contra algo, y la palabra corrupción significa la transgresión de un marco normativo por parte de uno o varios decisores con el objeto obtener una ventaja o ganancia para él o para otros a cambio de una contraprestación.

Por lo que el término anticorrupción puede englobar una serie de actos públicos como: las políticas públicas y programas, los cuales están dirigidos hacia el desarrollo de las llamadas "(actividades anticorrupción) cuyos objetos buscan: prevención, reducción, investigación, restricción, persecución, cumplimiento, enjuiciamiento, reparación o sanción de acto(s)u hecho(s) que transgredan un marco normativo, mediante el cual se conduce las actividades, entes, recursos u objetivos públicos" (Martínez, 2018, p. 25). Usualmente las denominadas actividades anticorrupción se ejecutan a través del denominado combate a la corrupción, el cual es comprendido como el "conjunto de esfuerzos orientados a construir condiciones y mecanismos institucionales (reglas, principios, procesos, rutinas) para prevenir, controlar y, en su caso, sancionar el uso indebido de los recursos públicos con fines privados (personales o grupales)" (Dussauge, 2010), p. 210.

\section{¿Qué es la Nueva Normalidad?}

La Nueva normalidad es una expresión del ámbito de la economía, finanzas y el comportamiento social en general acuñada para describir las nuevas condiciones financieras tras la crisis de 2008, las secuelas de la gran recesión y especialmente de la pandemia de enfermedad por el Coronavirus del 2019-2020 causada por el virus Sars.cov.2. Desde entonces, el término se ha utilizado en una variedad de contextos para dar a entender que algo que antes era anómalo ahora es común.

\section{Corrupción y Nueva Normalidad}

Uno de los muchos lastres que arrastra la sociedad mexicana es la corrupción gubernamental. De acuerdo a la Encuesta Nacional de Calidade Impacto Gubernamental (ENCIG), realizada por el INEGI, después de la inseguridad y la violencia, el segundo problema más importante del lugar donde viven las y los mexicanos es la corrupción.

El cinismo de los gobernantes sobre este problema que le cuesta 12,770 millones de pesos a la sociedad mexicana o un promedio de 3,822 pesos por persona, de acuerdo a la ENCIG 2019, llevó a un expresidente a declarar en un evento público que la corrupción era parte de la cultura mexicana y, por tanto, imposible de erradicar. La corrupción va desde el soborno a un policía para evadir una multa de tránsito, pagar para colgarse de la luz, las extorsiones de los municipios a los puestos ambulantes, la compra de medicinas cuya fecha de caducidad ya pasó, la adquisición de pizarrones electrónicos que no funcionan, el tráfico de influencias y la asignación de contratos millonarios de obra pública o servicios, que a pesar de que se licitan, acaban, sospechosamente, en las manos de los mismos de siempre. Dentro de los muchos retos y deudas que el gobierno mexicano tiene con su sociedad, que ha salido a relucir con la pandemia del COVID19 , es el combate a la corrupción, ya que su persistencia en el tiempo se debe a que no se sanciona y en caso de que se castigue las penas no son correspondientes al daño público y social que se hizo.

Son las consecuencias y efectos de actos de corrupción pasados los que han complicado en México aún más la crisis del COVID-19 y el regreso a la nueva normalidad. En materia de salud no se cuenta con la infraestructura adecuada o bien es y obsoleta y funciona parcialmente; asimismo no se tienen los insumos necesarios, como medicamentos o equipos de protección para el personal de salud debido a que los recursos públicos nunca llegaron. 
El regreso a la "nueva normalidad" o a las actividades productivas para que comience la reactivación de la economía es, otra vez, sumamente complejo debido a los engaños y falsificaciones que los empresarios han realizado en colusión con las respectivas autoridades gubernamentales para obtener los permisos para operar bajo condiciones laborales precarias e insalubres y en instalaciones peligrosas. En materia educativa, el problema es nuevamente la falta de infraestructura en las escuelas cuyas instalaciones no son dignas y, en ciertos lugares, no tienen siquiera acceso a un recurso tan indispensable como es el agua potable.

Las principales medidas de salud para eliminar al COVID-19 son el lavado frecuente de manos con agua y jabón y la sana distancia, pero ¿cómo sería posible llevarlas a cabo en escuelas con falta de agua y salones con sobre cupo, o en lugares de trabajo insalubres? La desconfianza entre las personas e instituciones, así como la incertidumbre de que todas las empresas cuenten con las políticas para garantizar el regreso seguro de los trabajadores a las actividades productivas, justifican el actuar cauteloso del gobierno para la reapertura económica y de las actividades sociales. Cuando pensemos en la corrupción no sólo hay que hacerlo en términos numéricos, sino también en las oportunidades de desarrollo que se acaban con la malversación de los recursos públicos.

\section{Política anticorrupción y política pública}

En los sistemas democráticos de hoy la solución a los problemas públicos se realiza a través de una serie de procesos políticos en los cuales intervienen una serie de actores interesados, a este tipo de mecanismo institucional de solución de problemas públicos se le conoce como políticas públicas. Exponen (Knoepfel, Larrue, Varone, Hinojosa, 2007) que las políticas públicas son "una concatenación de decisiones o de acciones, intencionalmente coherentes, tomadas por diferentes actores, públicos y ocasionalmente privados -cuyos recursos, nexos institucionales e intereses varían-, a fin de resolver de manera puntual un problema po- líticamente definido como colectivo" (p. 12). En este orden de ideas, el proceso político en el que se integran diversos actores con el fin de crear soluciones contra la corrupción definida como problema público, puede ser definida como una política anticorrupción, ya que según (UNODC, 2013), (Hussmann, 2007), (Hussmann y Hechler, 2008) y (COE, 2013) las políticas anticorrupción son un campo de la política pública, mediante el cual se desarrolla procesos dirigidos a la solución de los problemas públicos de corrupción y gobernabilidad a través del establecimiento de actividades anticorrupción.

De esta forma, el México de antes del año 2000 no produjo políticas públicas tendientes a combatir la corrupción ya que el problema fue definido únicamente en la agenda social y debido a la presión social proveniente de muchos sectores de la sociedad y academia éste fue colocado en la agenda pública, sólo para ser cooptado por la agenda gubernamental y ser definido como un problema de control interno de la Administración Pública Federal.

Este panorama cambio a partir del año 2000, con la denominada transición democrática, lo que permitió el desarrollo y autonomía de muchos entes públicos y el robustecimiento de la sociedad civil. El cambio motivó la exigencia de mecanismos para controlar la corrupción en el país y el surgimiento de nuevos canales institucionales para incidir en la definición de la corrupción como un problema público. Este objetivo se cumplió en el sexenio 2012-2018 mediante la denominada reforma anticorrupción, la cual desarrollo el primer marco institucional global en la materia: el Sistema Nacional Anticorrupción.

La construcción del Sistema Nacional Anticorrupción ha generado la puerta para que los actores sociales, académicos, políticos e institucionales desarrollen las políticas públicas tendientes a crear normas, agencias, proyectos y programas de concientización, prevención y educación contra la corrupción, ya que la" elaboración de las políticas anticorrupción no consiste en un proceso muy racional con 
objetivos fijos que los tecnócratas expertos controlen para alcanzar resultados previstos o declarados" (Hussmann y Hechler, 2008, p. 2).

\section{El Sistema Nacional Anticorrupción}

La sociedad mexicana del siglo XXI se encontró con la posibilidad de incidir en la construcción de las políticas públicas, producto del fortalecimiento de los entes autónomos, la descentralización de algunas atribuciones del poder ejecutivo y el desarrollo de vigoroso de una nueva sociedad civil. Este panorama generó la posibilidad de influir de forma directa en la policy network -policy community (comunidad política), la cual, a diferencia de los anteriores intentos realizados en el siglo XX, contó con una nutrida participación de policy stakeholders (sector implicado) en la construcción de la agenda y definiciones de los policy makers (tomadores de decisiones políticas) y por tanto en la propiedad de la nueva política pública en materia de combate a la corrupción.

En esta misma dirección señala (Makowski, 2016) que "la propiedad es la capacidad de manipular el significado, la definición del problema y gestionarlo, pero también es un conjunto de obligaciones y responsabilidades para la formulación de la política hacia el problema" (p. 57). De esta forma el desarrollo de una policy network más abierta marcaria "el inicio de un movimiento ciudadano, aun en marcha, que conjunta a instituciones académicas, organizaciones de empresarios y de la sociedad civil decididas a lograr una transformación en la manera de entender y combatir la corrupción en México" (Fierro, 2017), p. 95.

La transformación de la comunidad política interesada en combatir la corrupción, no sólo obedeció a las actuales exigencias sociales e institucionales en la materia, también se deben a un path dependence (camino de dependencia), mediante el cual las acciones realizadas por las organizaciones de la sociedad civil y recomendaciones de órganos supranacionales en los años 80 "provocaron que se formara un marco de actores, temas y acciones (camisa de fuerza) que el Gobierno mexi- cano incluyó paulatinamente y que delimitaron el desarrollo de sus acciones anticorrupción” (Martínez, 2018), p. 114.

En consecuencia, las acciones de los policy stakeholders lograron instituir una nueva realidad anticorrupción en el orden instituido, ya que según Berger y Luckman "institucionalización es un proceso en el cual ciertos actores crean una realidad que subsecuentemente se objetiviza, se toma como real, y es internalizada por otros" (Schvarstein, 1998), p. 292. Esto permite comprender porque el desenlace de la reforma anticorrupción terminó en la construcción de un sistema anticorrupción y no en otro órgano de combate al fenómeno, ya que otro tipo de institución no permitiría dirigir una atención integral del problema y no podría reunir múltiples sectores implicados bajo un solo techo.

Mediante la creación de un sistema de combate a la corrupción, se institucionalizó un orden lógico de entidades bajo una cabeza de integración colegiada, lo que permite generar un órgano de trabajo y contrapeso que permite prescindir de un órgano con hegemonía en la materia. Esto refleja la preocupación del sector social y académico por generar un ente cuya labor no pueda ser cooptada políticamente y cuyas facultades sean atendidas de forma íntegra por órganos especializados en la materia. De esta forma la creación de un sistema genera la posibilidad de contar con un ente de prevención que cuente con funciones de sanción y persecución.

A pesar de que el SNA es un ente multipropósito, las preocupaciones del sector implicado en la política anticorrupción causaron que se construyera un ente de prevención del fenómeno ya que bajo las clasificaciones de (Mea-gher, 2004), (De Sousa, 2009), y (OCDE, 2008) sobre Anti-Corruption Agencies ACAs (agencias anticorrupción) el sistema anticorrupción mexicano encaja en la descripción de un ente de prevención, ya que sus objetivos se dirigen a la concientización, la educación, el monitoreo y la coordinación de tareas, estrategias y políticas. Esto nos 
lleva a la pregunta ¿por qué se creó un ente anticorrupción bajo una estructura de sistema?

La respuesta no podría ser otra que la evidente desconfianza en las instituciones del Estado mexicano; sin embargo, la verdadera respuesta no es la crisis de confianza en las instituciones sino la crisis moral que vive la sociedad e instituciones mexicanas. Éste es el verdadero componente que lleva la comisión de actos corruptos y a la inoperancia de las instancias encargadas de sancionar los actos de corrupción. Dado que la estrategia de agencia única de combate a la corrupción requiere de y un liderazgo hegemónico y un amplio apoyo de los policy makers, la mayoría de los países solo desarrollan entes de combate a la corrupción alternativos, siempre y cuando las condiciones de gobernabilidad e integridad sean rebasadas:

En estas situaciones, puede haber un gran escándalo o, al menos, una percepción amplia de que las estructuras existentes han demostrado ser inadecuadas para prevenir o reprimir costosos errores éticos. Sin embargo, la profundidad de la crisis y la fortaleza del liderazgo son insuficientes para apoyar una centralización más robusta de las funciones anticorrupción. En otras palabras, las agencias judiciales y administrativas tradicionales conservan sus capacidades básicas y su legitimidad, mientras que se establecen estructuras adicionales para abordar las brechas, las debilidades y las nuevas oportunidades para la corrupción (Meagher, 2004, p. 36).

Bajo este argumento, la creación del Sistema Nacional Anticorrupción, como una agencia de tipo multi-agencias o modelo alternativo Meagher (2004), tiene mucho sentido si observamos los datos sobre confianza del Latinobarómetro y el World Values Survey (encuesta mundial de valores) WVS, en los cuales la confianza en las instituciones mexicanas y los valores de su sociedad van en declive. Por esta razón, el sector implicado o policy stakeholders buscaron la integración institucional de la sociedad civil organizada en la estructura del Sistema Nacional Anticorrupción, a través de la creación del

\section{Comité de Participación Ciudadana CPC} Mediante la inclusión del CPC se institucionaliza la participación de la sociedad civil organizada en el cuerpo colegiado del Sistema $\mathrm{Na-}$ cional Anticorrupción, generándose así un mecanismo de contrapeso frente a los órganos públicos que conforman el sistema. Este contrapeso surge de la evidente carga política que poseen los órganos que integran el cuerpo coordinado, en específico la Secretaría de la Función Pública, Fiscalía General de la República y la Auditoría Superior de la Federación, órganos que están sujetos bajo procesos de políticos que desarrollan los partidos y el Ejecutivo Federal.

A pesar de la evidente carga política, la estructura colegiada del SNA permite que las diversas entidades que comprenden al sistema generen contrapeso a la hora de coordinar sus tareas y así evitar el uso del entramado institucional con fines políticos. De acuerdo con (Martínez, 2018) las actividades anticorrupción bajo esquema de coordinación que desarrolla el SNA son: 1. Prevención (Secretaría de la Función Pública, Órganos Internos de Control y el Comité de Participación Ciudadana); 2. Control (Secretaría de la Función Pública y Auditoría Superior de la Federación); 3. Investigación y persecución (Secretaría de la Función Pública; Fiscalía General de la República y Auditoría Superior de la Federación); y 4. Sanción (Consejo de la Judicatura Federal y Tribunal Federal de Justicia Administrativa).

La creación del Sistema Nacional Anticorrupción SNA, no sólo fue un proceso legislativo o político, sino que supuso un proceso social mediante el cual la exigencia de los valores sociales e institucionales cambiaron el orden instituido y centralizado en posiciones objetivas con posiciones y decisiones técnicas, por un orden basado en la prevención, educación y concientización en el cual las instituciones formales (las leyes) y las instituciones infórmales (la moral, los valores, la ética) se entrelazan para enerar políticas y programas dirigidas a atender el problema corrupción, desde una pluralidad de enfoques y métodos. 


\section{CONCLUSIONES}

Como primera conclusión, se puede observar que los valores y la moral han servido como elementos en el mejoramiento de la condición social y protección de las prácticas e instituciones democráticas. Por lo tanto, su empleo no debe limitarse a la función de corregir de manera informal las conductas de la sociedad ni disminuir su capacidad de influencia en la toma de decisiones de los ciudadanos y los servidores públicos. Segundo, el desarrollo del Sistema Nacional Anticorrupción implicó en si una política anticorrupción, ya que en la corrupción fue definida como un problema público, se colocó en la agenda pública, se desarrolló la discusión con los actores de la policy community, se formuló la política (la solución al problema definido), se adoptó la decisión (se creó su norma y crearon los entes) y se implementó (se eligieron, crearon, coordinaron y dotaron de recursos lato sensu los órganos).

Tercero, con la implementación de la Sistema Nacional Anticorrupción se rompió el paradigma tradicional en el cual solo los policy makers son los únicos actores con voz y voto en la elaboración de las políticas públicas. De esta forma, cualquier persona o grupo a quien le afecte un problema público puede tomar parte en la solución, como lo es el caso de las políticas de seguridad y gobernabilidad, en las cuales las necesidades y la comunidad política son inmensas y difusas.

Cuarto, con la creación del Sistema Nacional Anticorrupción y en específico del Comité de Participación Ciudadana CPC, las políticas públicas en materia de combate a la corrupción adoptan un enfoque de prevención que solo la educación formativa (basada en valores y principios) puede generar nuevas soluciones a un problema tan arraigado en la sociedad mexicana.

Quinto, con el enfoque de prevención, se devuelve parte de interés hacia el estudio de las conductas y valores sociales que las políticas han olvidado por preferir enfoques económicos y racionales que omiten la esencia humana y mo- ralidad de las conductas sociales que rodean al problema corrupción.

\section{Referencias}

Aristóteles. (1222). Introducción. Virtudes Intelectuales para el Perfeccionamiento de los Actos Verdaderos, 15, 222.

(Bungue 2018). Recuperado del Diario el Pais https://elpais.com/autor/mario-bunge/

Alles 2008 recuperado del sitio o región de internet de: https://www.academia.edu/36640906/comportamiento_organizacional_martha_alles_pdf

Asped y López. (1999). 2.1 Hábitos. Marco Teórico, 2,7 .

(Sartre, 1994), p. 371. Ciencia y Sociedad, vol. XXXII, núm. 3, julio-septiembre, 2007

COE, (2013). Designing and Implementing Anti-corruption Policies, Directorate General Human Rights and Rule of Law Council of Europe.

Dussauge, M., (2010). Combate a la corrupción y rendición de cuentas: Avances, limitaciones, pendientes y retrocesos. En José Luis Méndez (coord.), Políticas Públicas (pp. 207-252). D.F., México: El Colegio de México, COLMEX.

Fierro Viesca Jacinto, 2017. p.95 ). El Federalismo Mexicano: Régimen Constitucional del Sistema Federal. México: Instituto Nacional de Administración Pública, INAP.

Garzón, E. (1997). Acerca del Concepto de Corrupción. En Laporta y Álvarez (coords.), en La Corrupción Política. Poder derecho y corrupción (pp. 39-69). Madrid, España: Alianza Editorial.

Giddens, A. (1999). Sociología. Madrid. Alianza. Recuperado de https://cmapspublic3.ihmc.us/rid=12 08893576750_474220728_4121/sociedad-definiciones.pdf

Gonzales, J. (2003). Corrupción, democracia y responsabilidad política. En Vásquez y Carbonell (coords.), Poder derecho y corrupción (pp. 63-80). Estado de México, México: Editorial Siglo XXI.

(Heidenheimer et al., 1989) extraído o recuperado de Sitio web: https://books.google.com.mx/ books?hl=es \&lr=\&id=49y50pzjAzAC \&oi =fnd \&p$\mathrm{g}=$ PA59\&dq=Heidenheimer+et+al.,+1989)\&ots=Wfkoh8fBdg \& sig $=$ hr 38f2 oVryqXq 4BFls0 O GAB GaL4\#v=onepage \& $\mathrm{q}=$ Heidenheimer\%20et\%20 al.\%2C\%201989)\&f=false

Hussmann, K. (2007). La construcción de políticas anticorrupción en la práctica: ¿Qué se puede aprender para la implementación del Artículo 5 de la CNUCC? Informe comprensivo sobre seis estudios de caso. Bergen, Noruega: CMI- Chr. Michelsen Institute.

Hussmann, Ky Hannes, H. (2008). La construcción de políticas anticorrupción en la práctica: Implicaciones de la implementación de la CNUCC, U4Brief, núm. 
19. Bergen, Noruega: CMI- Chr. Michelsen Institute.

Jorge, J. (2010). Cultura Politica y Democracia en Argentina. Buenos Aires: EDULP. (pp. 18) Subsecretaría de Educación Básica. (2019). SISTEMA EDUCATIVO MEXICANO. 24 de marzo de 2019, de Secretaría de Educación Pública [MX] Sitio web: https:// www.mexterior.sep.gob.mx/sisedMEX.html

Kelsen H. (2000). ¿Qué es la justicia? Recuperado de https://es.scribd.com/doc/122793204/Hans-Kelsen-Que-Es-La-Justicia-pdf

Klitgaard Robert, Maclean Abaroa Ronald, H. Parris Lindsey, (2000). Corrupt Cities: A Practical Guide to Cure and Prevention. World Bank, ICS Press

Knoepfel Peter, Larrue Corinne, Varone Frédéric, Hinojosa Dieck Miriam, (2007). Hacia un modelo de análisis de políticas públicas operativo. Un enfoque basado en los actores, sus recursos y las instituciones, Revista Ciencia Política, Vol. 2 (3), 6-29.

Leys Colin, (1965). What is the Problem about Corruption? The Journal of Modern African Studies, vol. 3 (2), 215-230.

Makowski Grzegorz, (2016). Anti-corruption Agencies - Silver Bullet Against Corruption or Fifth Wheel to a Coach? Analysis from the Perspective of the Constructivist Theory of Social Problems. Journal Studia z Polityki Publicznej, vol. 3: (11), 55 - 78.

Martínez, E, (2018). El desarrollo del combate a la corrupción y la agencia anticorrupción en México (Tesis de maestría). El Colegio de San Luis, San Luis Potosí, México.

Meagher Patrick, (2004). Anti-Corruption Agencies: a review of experience, Center for Institutional Reform and the informal Sector. USA: University of Maryland, Paper No. 04/02.

Morales, J. (2017). Descartes: filósofo de la moral. Estudios de filosofía, (55), 11-29. Doi: 10.17533/udea. ef.n55a02
Nunes, P. (2015). Concepto de Jerarquía. Recuperado el 24/03/2019 desde http://knoow.net/es/cieeconcom/gestion/jerarquia/.

OCDE, (2008), Specialised Anti-Corruption Institutions: Review of models, OECD publications.

Posadas Ramírez, J. (2014). Ontología y Lenguaje de la Realidad Social. Obtenido el 24, marzo, 2019 recuperado desde https://scielo.conicyt.cl/scielo.php?script=sci_arttext\&pid=S0717-554X2014000200003

(Parker, 2001). Ciencia y Sociedad, vol. XXXII, núm. 3, julio-septiembre, 2007

(Platón, 2013) recuperado de https://www.redalyc. org/pdf/414/41431644008.pdf

RAE, (2019). vigesimotercera edición. Definición de "corrupción", obtenida de: https://dle.rae.es/?i$\mathrm{d}=$ BOdY4l3

Schvarstein, L. (1998). Diseño de organizaciones: Tensiones y paradojas. Argentina: Paidós.

De Sousa, L. (2009). Anti-Corruption Agencies: Between Empowerment and Irrelevance. Editor European University Institute.

UNODC, (2013). Hacia una Primera Reflexión sobre Políticas Anticorrupción: Algunos Insumos para la Implementación de la Convención de las Naciones Unidas contra la Corrupción desde la Experiencia de América Latina, Oficina Regional de UNODC para Centroamérica y el Caribe (ROPAN). Panamá.

(Weber 1979) Extraido oportunamente de la region de internet siguiente: http://www.politicascti.net/index.php?option=com_docman\&task=doc_view\&gi$\mathrm{d}=43$ \&Itemid $=36 \&$ lang $=$ es

Valladares, A. M. (2008). La familia. Una mirada desde la Psicología. Revista Electrónica de las Ciencias Médicas en Cienfuegos, 6 (1), 4-13. Recuperado de file://C:/Users/JORGE/Downloads/402-9057-1-PB. pdf 